\title{
(C) OPEN ACCESS \\ The development of a tongue assessment tool to assist with tongue-tie identification
}

\author{
Jenny Ingram, ${ }_{1}^{1}$ Debbie Johnson, ${ }^{1}$ Marion Copeland, ${ }^{2}$ Cathy Churchill, ${ }^{3}$ Hazel Taylor, ${ }^{4}$ \\ Alan Emond ${ }^{1}$
}

${ }^{1}$ School of Social and Community Medicine, University of Bristol, Bristol, UK ${ }^{2}$ Midwifery, North Bristol NHS Trust, Southmead Hospital, Bristol, UK

${ }^{3}$ Neonatal Intensive Care Unit, North Bristol NHS Trust, Southmead Hospital, Bristol, UK

${ }^{4}$ R\&l, University Hospitals Bristol NHS Foundation Trust, Bristol, UK

\section{Correspondence to}

Dr Jenny Ingram, Centre for Child and Adolescent Health, School of Social and Community Medicine, University of Bristol, Oakfield House, Bristol BS8 2BN, UK jenny.ingram@bristol.ac.uk

Received 12 September 2014 Revised 9 March 2015 Accepted 22 March 2015 Published Online First 15 April 2015

\section{ABSTRACT}

Aim To produce a simple tool with good transferability to provide a consistent assessment of tongue appearance and function in infants with tongue-tie. Methods The Bristol Tongue Assessment Tool (BTAT) was developed based on clinical practice and with reference to the Hazelbaker Assessment Tool for Lingual Frenulum Function (ATLFF). This paper documents 224 tongue assessments using the BTAT. There were 126 tongue assessments recorded using the BTAT and ATLFF tools to facilitate comparisons between them. Paired BTAT assessments were obtained from eight midwives who were using the new assessment tool.

Results There was acceptable internal reliability for the four-item BTAT (Cronbach's $\alpha=0.708$ ) and the eight midwives who used it showed good correlation in the consistency of its use $(I C C=0.760)$. The BTAT showed a strong and significant correlation (0.89) with the ATLFF, indicating that the simpler BTAT could be used in place of the more detailed assessment tool to score the extent of a tongue-tie. Midwives found it quick and easy to use and felt that it would be easy to teach to others.

Conclusions The BTAT provides an objective, clear and simple measure of the severity of a tongue-tie, to inform selection of infants for frenotomy and to monitor the effect of the procedure.

\section{INTRODUCTION}

There is increasing evidence that the presence of a tongue-tie (ankyloglossia) in an infant may lead to breastfeeding difficulties. Feeding difficulties (both breast and bottle) have been reported in 25\%-44\% of infants with tongue-tie. ${ }^{1-4}$ Difficulties included inability to feed continuously, poor attachment, unsettled infants with poor weight gain and maternal nipple trauma.

Dividing the tongue-tie, frenotomy, is described as a simple procedure in the young infant which can be performed without an anaesthetic and having few complications. ${ }^{5}$ Historically, it was once an accepted procedure that appears to have fallen out of favour in the late 20th Century. ${ }^{6}$ Currently, the practice of frenotomy in the UK is increasing rapidly in response to greater recognition of the importance of the tongue in successful breastfeeding and despite many health professionals being unconvinced of the need for or efficacy of the procedure. The result is a piecemeal and inequitable service provision across the country, at odds with the ethos of the National Health Service.

The reported incidence of tongue-tie ranges in worldwide studies from $3 \%$ to $16 \% .^{12} 78$ This variation may be due, in part, to a lack of

\section{What is already known on this topic?}

The incidence of tongue-tie (ankyloglossia) ranges from $3 \%$ to $16 \%$ worldwide and the presence of a tongue-tie in an infant may lead to breastfeeding difficulties.

- There is inequitable frenotomy service provision across the UK due to a lack of evidence and health professionals being unconvinced of its need or efficacy.

- An objective assessment tool is needed to inform optimum timing of frenotomy and level of severity that would benefit most from the procedure.

\section{What this study adds?}

The Bristol Tongue Assessment Tool (BTAT) provides an objective, clear and simple indication of the severity of the tongue-tie.

- The BTAT showed good internal reliability, and correlated well with the Hazelbaker Assessment Tool for Lingual Frenulum Function (short form).

agreement about when a frenulum is normal in appearance versus tongue tied. A common definition of tongue-tie includes a tight frenulum with inability of the tongue to protrude beyond the lower incisors ${ }^{5}$ or the lower gum (http:// www.unicef.org.uk/BabyFriendly/Parents/Problems/ Tongue-Tie). National Institute for Health and Care Excellence $^{5}$ describes the degree of tongue-tie as varying from mild to severe, with breastfeeding difficulties arising from the inability of the infant to latch and suck effectively. Hazelbaker ${ }^{9}$ stresses the need for the definition to include assessment of tongue function, and has developed the Assessment Tool for Lingual Frenulum Function (ATLFF), which she uses in her own detailed breastfeeding assessments.

As an objective measure of tongue-tie, the ATLFF comprises five appearance and seven function criteria. ${ }^{9}$ It has been used as an assessment tool in several research studies ${ }^{7}$ including the recent Bristol Tongue-tie Trial ${ }^{10}$ where it was used in both the full ${ }^{9}$ and the shortened form. ${ }^{11}$ The shortened form was suggested by Amir $e t a l^{11}$ and used only three of the function items. However, Hazelbaker stresses that the full measure should be 
used as part of a detailed feeding assessment, not simply to determine the severity of a tongue-tie by its appearance, and that the importance of assessing tongue function when deciding upon possible frenotomy must not be overlooked. Ngerncham et $a l^{8}$ found the ATLFF tool to be very comprehensive, but difficult to use and not suitable as a screening tool in a busy hospital setting. Similarly, the study by Madlon-Kay et $a l^{12}$ reported that they found it difficult to classify many babies using the tool.

The Bristol Tongue-Tie trial ${ }^{10}$ required a method of quantifying the severity of tongue-tie, as only those in the 'mild to moderate' range were eligible to be included. Two experienced lactation consultants spent 3 months before the trial commenced gaining confidence using the ATLFF tool in the clinical setting and ensuring consistency. When the trial started, the lactation consultants continued to carry out joint assessments whenever possible. Two new midwife assessors joined during the trial to help with recruitment, but it became apparent that the ATLFF was not an ideal screening tool for a study that involved several different assessors. In particular, assessors found it difficult to achieve good agreement on the length and elasticity of the frenulum, which has also been found by others, ${ }^{12}$ but tongue appearance and attachment of the frenulum to the tongue and ridge were easier to agree on.

A recent systematic review identified studies that reported the effects of frenotomy on breastfeeding and the objective scoring systems used in the studies. ${ }^{13}$ Webb et al concluded that tonguetie division improves many aspects of breastfeeding for most newborns and their mothers. They recommended that validated measures of the severity of ankyloglossia and breastfeeding outcomes should be developed and studies to elucidate the definition and significance of ankyloglossia with regard to the proper timing of the corrective procedure were needed. ${ }^{13}$

We aimed to produce a simple assessment tool with good transferability to provide consistent assessment of tongue appearance and function for infants with tongue-tie.

\section{METHODS}

\section{Development, analysis and validation of the Bristol Tongue} Assessment Tool

Three midwife researchers identified the four most important aspects of newborn tongue assessment based on their clinical experience and knowledge of other scoring tools, ${ }^{13}$ including the ATLFF. From these a new assessment tool was developed: the Bristol Tongue Assessment Tool (BTAT, as shown in table 1). The tool was developed and introduced as a second assessment part way through recruiting babies into the feasibility trial. ${ }^{10}$ The midwives who performed frenotomy within the hospital were trained together to use the tool at a study day led by the midwife researchers who developed it. They were encouraged to use it whenever they assessed a baby for tongue-tie.

The tool elements are: (see table 1 for BTAT)

1. Tongue tip appearance was selected as for many this is one of the primary ways of assessing a tongue-tie. It is often noticed by parents and so can be useful in explaining the presence of tongue-tie to them.

2. Attachment to the lower gum ridge allows the clinician to assess the presence of tongue-tie in cases where the appearance may not be obvious. This was selected rather than attachment of frenulum to the tongue as attachment to the tongue is usually reflected in the appearance of the tongue with mouth wide.

3. The lift of the tongue is also easy to see as long as the infant is awake and is ideally viewed when the infant is crying. This is the item that has proved most difficult to teach and does require an awareness of normal lift in the tongue of a newborn infant. In a sub-mucosal tongue-tie, the tongue may appear to lift when it is actually curling backwards and it is this subtlety that can take longer to recognise.

4. Protrusion of the tongue. If the infant is asleep and the assessor is unable to elicit tongue protrusion, the parents will usually be aware of how far their baby can protrude its tongue. Protrusion can also be the first improvement that parents notice following frenotomy.

The scores for the four items are summed and can range from 0 to 8 . Scores of $0-3$ indicate more severe reduction of tongue function.

This paper documents 224 tongue assessments, 22 of which were from infants included in the feasibility trial ${ }^{10}$ and the remainder were from term infants $(37+$ weeks gestation) with feeding difficulties but not recruited during the trial period and for 5 months following the completion of trial recruitment, between July 2012 and November 2013. Breastfeeding difficulties were assessed using a recently validated tool, the Bristol Breastfeeding Assessment Tool $^{14}$ and clinical judgement. 126 tongue assessments were recorded using both BTAT and ATLFF (short form) scores to facilitate comparisons between the tools. Paired BTAT assessments were obtained from two infant feeding specialist researchers plus six midwives who had been taught the new assessment tool on the study day. These midwives routinely assessed tongue-tie and performed frenotomy within the hospital where the feasibility study was conducted. For each 'paired assessment', a research infant feeding specialist plus one other midwife examined the tongue independently, logging their observations on the BTAT. Each was 'blind' to the other's assessment until both were completed. To facilitate learning, they then discussed their interpretation of the scoring but no changes were made to their original scores. All statistical analysis was carried out using the statistical package SPSS. The dimensionality of the BTAT scale was examined using Factor Analysis. Cronbach's $\alpha,{ }^{15}$ as a measure of internal consistency, was used to estimate the reliability of the items within the BTAT. Pearson's correlation coefficient was used to measure the strength of the association between the BTAT and ATLFF.

An intraclass correlation coefficient was used to assess the consistency in BTAT scores obtained from pairs of assessors (two midwives from those assessing tongue-tie). Eight different

Table 1 Bristol Tongue Assessment Tool (BTAT)

\begin{tabular}{|c|c|c|c|c|}
\hline & 0 & 1 & 2 & Score \\
\hline Tongue tip appearance & Heart shaped & Slight cleft/notched & Rounded & \\
\hline Attachment of frenulum to lower gum ridge & Attached at top of gum ridge & Attached to inner aspect of gum & Attached to floor of mouth & \\
\hline Lift of tongue with mouth wide (crying) & Minimal tongue lift & Edges only to mid-mouth & Full tongue lift to mid-mouth & \\
\hline Protrusion of tongue & Tip stays behind gum & Tip over gum & Tip can extend over lower lip & \\
\hline
\end{tabular}


Table 2 Internal consistency and correlations for BTAT and Hazelbaker Assessment Tool for Lingual Frenulum Function (ATLFF)

\begin{tabular}{|c|c|c|c|c|}
\hline & $\mathbf{n}$ & $\begin{array}{l}\text { Cronbach's alpha } \\
(95 \% \mathrm{Cl})\end{array}$ & $\begin{array}{l}\text { Correlation } \\
(95 \% \mathrm{Cl})\end{array}$ & $\mathrm{p}$ Values \\
\hline All BTATs & 224 & 0.708 (0.641 to 0.766$)$ & & \\
\hline $\begin{array}{l}\text { BTATs for } 8 \\
\text { assessors }\end{array}$ & 33 & & $\begin{array}{l}\text { ICC: } 0.760 \\
\text { (0.566 to } 0.874)\end{array}$ & $<0.001$ \\
\hline $\begin{array}{l}\text { BTAT with } \\
\text { ATLFF }\end{array}$ & 126 & & $\begin{array}{l}\text { Pearson: } 0.889 \\
\text { (0.846 to } 0.921)\end{array}$ & $<0.001$ \\
\hline
\end{tabular}

assessors were involved in these comparisons although each infant was only assessed once by two different assessors.

Midwives were interviewed to explore their views of using the tool in their clinical practice. A short telephone interview was conducted by the trial manager or senior researcher, which was recorded and transcribed. Simple thematic analysis using an inductive approach was used to scrutinise the data to identify and analyse patterns across the dataset. Following repeated reading of the short transcripts, codes were generated and built into themes. ${ }^{16}$ Two researchers coded the interviews and agreed the themes. Final themes were discussed within the research team to achieve a coding consensus.

Comments made by the midwives are reported to illustrate views of the wider group of midwives and not just those who were involved in developing the tool.

The study was approved by the Central Bristol Research Ethics Committee (South West) (11/SW/0087). Mothers gave written consent for their data to be included and midwives gave verbal consent for the interviews to be recorded and quotes reported.

\section{RESULTS}

Infants assessed for the study had a mean age of 17 days (median 10 days, range 0-79); those within the trial had a mean age of 6 days (median 6 days, range $0-13$ ) and non-trial infants had a mean age of 18 days (median 12 days, range $0-79$ ).

BTAT scores were recorded for 224 assessments and ATLFF for 126. BTAT assessments with two midwives together were completed for 33 infants. The internal consistency and correlations are shown in table 2 and figure 1.

Factor analysis demonstrated that the items of the BTAT scale are unidimensional. There was acceptable internal reliability for the four-item BTAT (Cronbach's $\alpha=0.708$ ), and the eight midwives who used it showed good correlation in the consistency of its use when comparing with paired assessments carried out on the same infant $(\mathrm{ICC}=0.760)$.

The BTAT showed a strong and significant correlation (0.89) with the ATLFF, indicating that the simpler BTAT could be used in place of the more detailed assessment tool.

\section{Interviews with midwives}

Seven midwives were interviewed and their comments illustrate that they all found the elements of the tool self-explanatory and easy and quick to use. None had yet taught it to other midwives as all those who routinely performed frenotomy had attended the study day; however, they felt confident that they would be able to cascade this training. Themes around 'ease of use to confirm the diagnosis', 'it helps with explanations to parents' and 'teaching it to others' are illustrated with quotes from the midwives.

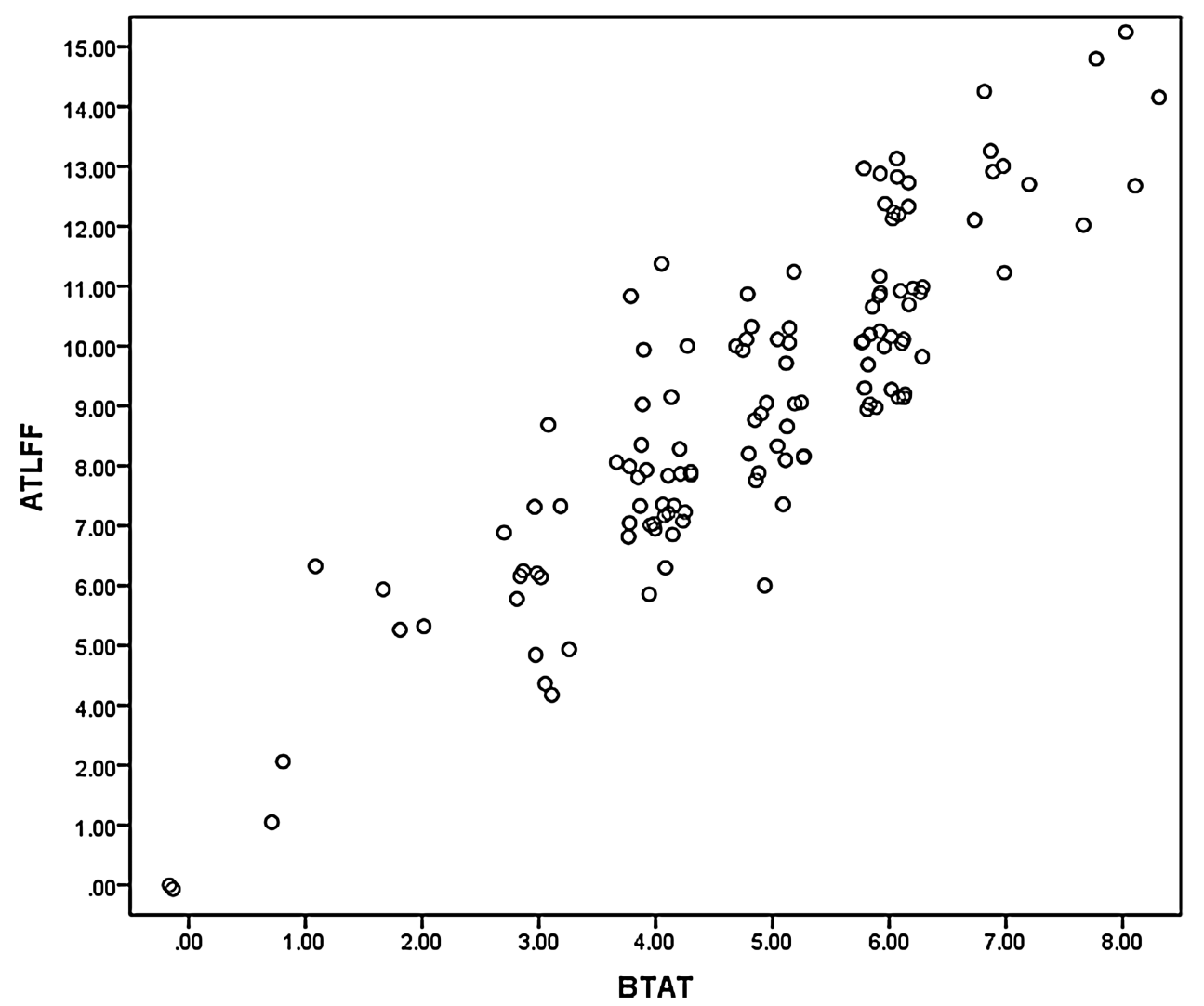

Figure 1 Correlation between Bristol Tongue Assessment Tool (BTAT) and Assessment Tool for Lingual Frenulum Function (ATLFF) scores for 126 assessments. 
Ease of use to confirm the diagnosis

It is much easier to use than the ATLFF, quicker and less equivocal. The ATLFF had some subjective points but this tool is easier and I will continue to use it in my practice. It hasn't changed practice but confirmed my thoughts about a tongue-tie. It makes the assessment much more simple and confirms whether the frenotomy needs doing. MW\#3

I have used it in practice and it helps to identify whether the tongue-tie is significant. It's quick and easy to use and helps the decision about whether to do division. MW\#2

The Bristol one is better and much clearer to follow than ATLFF. It is very relevant and clear and I started using it straight after the study day. I use the scoring system to confirm what I'm thinking and it helps with the decision to do frenotomy. It's an easy tool to use and it allows us to see the severity of the tongue-tie in a more formal way. It's not complicated at all and only takes 2 seconds to do. MW\#6

It backs up my clinical decision. MW\#7

\section{It helps with explanations to parents}

It definitely helps in explanations to parents-you can describe what is happening and tell them what the pointers are that we are looking for and if your baby doesn't have these then the tongue-tie is not serious. It's a very useful tool. MW\#2

I would use it to feed back information to the parents if I think it is affecting feeding. MW\#5

Sometimes mothers decide not to have them (frenotomy) done and the score helps that decision. MW\#7

\section{Teaching it to others}

It would be quite easy to teach to others. We all learnt together -we looked at the same baby and scored the tongue-tie and came up with similar scores. MW\#2

We learnt it at the study day with photos and discussed what the different items meant. I haven't taught it to anyone else but it would be easy to do. MW\#5

\section{DISCUSSION}

A concise and simple tongue assessment tool, the BTAT, was developed, based on clinical experience and with reference to the Hazelbaker ATLFF. Midwives found the BTAT easy to use and it was easily transferrable between midwives to provide consistent assessment of infants with tongue-tie identified in the early weeks. We suggest that the BTAT could be used to assess the incidence of tongue-tie in newborn infants as well as objectively assessing severity in research studies investigating the impact of frenotomy.

Limitations of our study include the fairly small number of multiple comparisons between midwives, but these are similar in number to those reported by others in validating infant screening tools. ${ }^{1417}$ The relationships between BTAT and breastfeeding duration and exclusivity were not explored in this study as the tool was mainly used to assess severity of tongue-tie. This relationship should be explored in further research studies as our results on breastfeeding duration were limited to those who had data collected within the frenotomy trial.

The debate continues about whether infants with tongue-tie require it to be divided, but recent commentaries ${ }^{18-20}$ and previous reviews ${ }^{3} 21$ have highlighted the need for frenotomy for infants experiencing breastfeeding difficulties. Woolridge ${ }^{18}$ emphasises the importance of a freely flexible and extendable tongue in the role of breastfeeding and efficient removal of milk from the breast, based on his ultrasound studies on infants' breastfeeding ${ }^{22}$ and studies by Geddes et al. $^{23}$ Reviews by Segal et $a l^{3}$ and Edmunds $e t a l^{21}$ both concluded that there are significant benefits of frenotomy for mothers and infants and that not offering a treatment that has shown to be beneficial would be unethical.

The commentary by Bowley and Arul ${ }^{19}$ concludes that around $80 \%$ of infants will respond positively to frenotomy with a low risk of harm. Those performing frenotomy should have received appropriate training, and clinical and ongoing lactation support are important. Assessment and division of tongue-tie for selected infants should be part of usual care which supports the initiation and maintenance of exclusive breastfeeding. Lawson ${ }^{20}$ highlights the need for more research to ensure that the correct infants are treated at the right time and that fashion does not dictate unnecessary interventions. Nationally, there should be universal provision for prompt and easy access to treatment for all infants whose feeding is compromised.

In conclusion, infants with tongue-tie who are experiencing difficulties with breastfeeding should be referred for assessment and lactation support. The BTAT provides an objective, clear and simple indication of the severity of the tongue-tie. Further research is needed to explore the optimum timing of the procedure and level of severity that would benefit most from frenotomy.

Acknowledgements We thank the mothers who took part in the trial and those who also provided data; the midwives and the lactation support team for their help in recruiting and supporting breastfeeding mothers. We also thank Dr Alison Hazelbaker for her comments and discussion around tongue-tie assessment.

Contributors Jl designed the study, was a member of the Bristol Tongue-Tie Trial management committee, supervised data collection, undertook the qualitative interviews and contributed to analysis and data interpretation. She wrote the early drafts of the paper and approved the final version of the manuscript. DJ was the trial manager and a member of the trial management committee. She collected outcome data, undertook some interviews and contributed to data interpretation. She contributed to the early drafts of the paper and approved the final version of the manuscript. MC was an expert member of the trial management committee. She assessed women for eligibility for the trial, undertook frenotomies and collected data and contributed to data interpretation. She contributed to the early drafts of the paper and approved the final version of the manuscript. CC was a member of the trial management committee. She assessed women for eligibility for the trial, undertook frenotomies and collected data and contributed to data interpretation. She approved the final version of the manuscript. HT undertook data analysis, contributed to data interpretation and approved the final version of the manuscript. AE was the local PI on the Bristol Tongue Tie Trial, contributed to data interpretation and approved the final version of the manuscript.

Funding This independent research was funded by the National Institute for Health Research (NIHR) under its Research for Patient Benefit (RfPB) Programme (Grant Reference Number PB-PG-0110-21019). The views expressed are those of the authors and not necessarily those of the NHS, the NIHR or the Department of Health. Only a small proportion of the infants in this study were in the RCT. The trial registration number for those infants is ISRCTN 73554751.

\section{Competing interests None.}

Ethics approval Central Bristol Research Ethics Committee (South West).

Provenance and peer review Not commissioned; externally peer reviewed.

Open Access This is an Open Access article distributed in accordance with the Creative Commons Attribution Non Commercial (CC BY-NC 4.0) license, which permits others to distribute, remix, adapt, build upon this work non-commercially, and license their derivative works on different terms, provided the original work is properly cited and the use is non-commercial. See: http://creativecommons.org/ licenses/by-nc/4.0/

\section{REFERENCES}

1 Messner AH, Lalakea ML, Aby J, et al. Ankyloglossia: incidence and associated feeding difficulties. Arch Otolaryngol Head Neck Surg 2000;126:36-9.

2 Ricke LA, Baker NJ, Madlon-Kay DJ, et al. Newborn tongue-tie: prevalence and effect on breast-feeding. J Am Board Fam Pract 2005;18:1-7. 


\section{Original article}

3 Segal L, Stephenson R, Dawes M, et al. Prevalence, diagnosis and treatment of ankyloglossia. Methodologic Review. Can Fam Physician 2007;53:1027-33.

4 Griffiths DM. Do tongue ties affect breastfeeding? J Hum Lact 2004;20:409-14.

5 NICE. Division of ankyloglossia (tongue-tie) for breastfeeding. Interventional procedures guidance [IPG 149]. December 2005. http://www.nice.org.uk/Guidance/IPG149

6 Dunn PM. Bridled babies: a history of tongue tie. Proc Bristol Medico Historical Soc 1995-1999;3:15-23.

7 Ballard JL, Auer CE, Khoury JC. Ankyloglossia: assessment, incidence, and effect of frenuloplasty on the breastfeeding dyad. Pediatrics 2002;110:e63.

8 Ngerncham S, Laohapensang M, Wongvisutdhi T, et al. Lingual frenulum and effect on breastfeeding in Thai newborn infants. Paediatr Int Child Health 2013;33:86-90.

9 Hazelbaker AK. Tongue-tie morphogenesis, impact, assessment and treatment. Columbus, Ohio: Aidan \& Eva Press, 2010.

10 Emond A, Ingram J, Johnson D, et al. Randomised controlled trial of early frenotomy in breastfed infants with mild-moderate tongue-tie. Arch Dis Child Fetal Neonatal Ed 2014;99:F189-95.

11 Amir LH, James JP, Donath SM. Reliability of the Hazelbaker assessment tool for lingual frenulum function. Int Breastfeed J 2006;1:3.

12 Madlon-Kay DJ, Ricke LA, Baker NJ, et al. Case series of 148 tongue-tied newborn babies evaluated with the assessment tool for lingual frenulum function. Midwifery 2008;24:353-7.

13 Webb AN, Hao W, Hong P. The effect of tongue-tie division on breastfeeding and speech articulation: a systematic review. Int J Pediatr Otorhinolaryngol 2013;77:635-46.
14 Ingram J, Johnson D, Copeland M, et al. The development of a new breastfeeding assessment tool and the relationship with breastfeeding self-efficacy. Midwifery 2015;31:132-7.

15 Streiner DL, Norman GR. Health measurement scales: a practical guide to their development and use. Oxford University Press, 1988

16 Silverman D. Doing qualitative research: a practical handbook. London: Sage publications, 2000.

17 Riordan J, Koehn M. Reliability and validity testing of three breastfeeding assessment tools. JOGNN 1997;26:181-7.

18 Woolridge M. Evidence that a short frenulum hampers the establishment of breastfeeding. Paediatr Int Child Health 2013;33:59-60.

19 Bowley DM, Arul GS. Fifteen-minute consultation on the infant with a tongue-tie. Arch Dis Child Educ Pract Ed 2014;99:127-9.

20 Lawson A. To snip or not to snip: the dilemmas of tongue-tie. Arch Dis Child Fetal Neonatal Ed 2014;99:178-9.

21 Edmunds J, Miles S, Fulbrook P. Tongue-tie and breastfeeding: a review of the literature. Breastfeed Rev 2011;19:19-26.

22 Monaci G, Woolridge M. Ultrasound video analysis for understanding infant breastfeeding. International Congress of Image Processing (ICIP). Brussels: IEEE, 2011. http://ieeexplore.ieee.org/stamp/stamp.jsp?tp5\&arnumber56115802\& isnumber 56115588

23 Geddes DT, Langton DB, Gollow I, et al. Frenulotomy for breastfeeding infants with ankyloglossia: effect on milk removal and sucking mechanism as imaged by ultrasound. Pediatrics 2008;122:e188-94. 